\title{
Hypothalamic Dynamics of CD26 Protein in Obese and Fasted Rats
}

\author{
Rafaela F Alpontia, b, Maria I Nogueirac ${ }^{\mathrm{c}}$, Paulo F Silveira ${ }^{\mathrm{a}, \mathrm{d}}$
}

\begin{abstract}
Background: Impaired control of brain functions by CD26 alterations and sensing of unbalanced metabolism could be related in obese and fasting rats. This study aims to search the hypothalamic regions targeted by the alterations of CD26 and cellular activity (Fos) in monosodium glutamate obese (MSG) and food deprived (FD) rats.
\end{abstract}

Methods: The patterns of distribution of immunoreactivity (ir) of CD26 and Fos were quantified in the hypothalamus.

Results: Compared to control, the CD26-ir increased in the supraoptic and retrochiasmatic nuclei of MSG. CD26-ir and Fos-ir concomitantly decreased in the dorsomedial and retrochiasmatic nuclei of MSG-FD, the paraventricular nucleus of MSG and MSG-FD and in the supraoptic nucleus of FD and MSG-FD, while they exhibited opposite changes in the retrochiasmatic nucleus of FD and MSG, the arcuate and periventricular nuclei of FD, MSG and MSG-FD, the paraventricular and dorsomedial nuclei of FD and the supraoptic nucleus of MSG.

Conclusions: The data provide clear evidences for regional specific alterations of CD26 together with altered cellular activity in the hypothalamus of MSG and FD animals and demonstrate that regulation of CD26 is a hallmark of hypothalamic exposure to metabolic challenges.

Manuscript accepted for publication May 26, 2011

${ }^{a}$ Laboratory of Pharmacology, Butantan Institute, Av. Vital Brasil, 1500, 05503-900 Sao Paulo, SP, Brazil

${ }^{\mathrm{b}}$ Department of Physiology, Biociencias Institute, University of Sao

Paulo, Brazil

${ }^{\mathrm{c}}$ Department of Neurosciences and Behavior, University of Sao Paulo, 05508-030 Sao Paulo, SP, Brazil

${ }^{\mathrm{d}}$ Corresponding author: Paulo F Silveira,

Email: pefesil@butantan.gov.br

doi:10.4021/jem24w
Keywords: CD26; Fos; Cellular activity; Obesity; Glutamate; Fasting; Brain

\section{Introduction}

The $\mathrm{T}$ cell marker CD26 is a multifunctional cell surface glycoprotein known to be induced upon $\mathrm{T}$ cell activation [1]. Investigations on inflammation and autoimmunity have pointed out a complex role for CD26 as an inhibitory leukocyte surface receptor that can act as a target for ligands blocking enzymatic activity and inhibiting $T$ cell activation $[2,3]$. The CD26 molecule functions as the cell membrane-associated dipeptidyl peptidase (DPP) IV (EC 3.4.14.5, gene: 3q21), an ectopeptidase capable of cleaving $\mathrm{N}$-terminal dipeptides from polypeptides with either proline or alanine residues at the penultimate position $[4,5]$. Among the substrates of the canonical CD26/DPPIV are glucagon-like peptides (GLP) types 1 and 2, glucose-dependent insulinotropic polypeptide or gastric inhibitor peptide (GIP), peptide YY 1-36 (PYY), neuropeptide Y 1-36 (NPY), endorphin-2, dynorphin, substance $\mathrm{P}$, growth hormone releasing hormone (GHRH) [413], CCL5 (RANTES), factor-1 derived from stroma and chemokine derived from macrophages [14]. Investigations on diabetes mellitus have pointed out a role for CD26/DPPIV mainly on the hydrolysis of GLP-1 $[15,16]$. Receptors of GLP-1 and GIP have been found in the brain [17], where their effects are beginning to be understood and their relevance to disorders of nutrition and aging is being exploited. Furthermore, CD26/DPPIV has been associated with an effector phenotype and is markedly elevated in the human multiple sclerosis [18]. However, whether CD26/DPPIV and cellular activation in the central nervous system (CNS) could be associated with obesity and nutritional status remains a critical challenge to elucidate. Recent studies suggested that altered gene expression and catalytic activity of CD26/DPPIV in plasma [19] and hypothalamus (HT) [20] occur in monosodium glutamate obese (MSG) and food deprived (FD) rat models. The focus of this study was to examine the pattern of regional distribution of $\mathrm{CD} 26$ and its relation to activated cells (Fos) in the HT of MSG and FD rats. 


\section{Materials and Methods}

\section{Animals}

Immediately after birth, male Wistar rats were housed with a lactating female in a polypropylene box (inside length $\mathrm{x}$ width $\mathrm{x}$ height $=56 \mathrm{~cm} \times 35 \mathrm{~cm} \times 19 \mathrm{~cm}$ ), with food (commercially available ration Nuvilab CR-1, Nuvital, Brazil; composed of $22 \%$ protein, $55 \%$ carbohydrate, $4 \%$ lipids, $8 \%$ fibers, $10 \%$ vitamins and minerals; total of $3 \mathrm{kcal} / \mathrm{g}$ ) and tap water ad libitum, in a ventilated container (Alesco Ind. Com Ltda, Brazil), with a controlled temperature $\left(24 \pm 2{ }^{\circ} \mathrm{C}\right)$, relative humidity $(65 \pm 1 \%)$ and $12: 12 \mathrm{~h}$ light/dark photoperiod (lights on at 6:00 a.m.). Twenty-four hours after birth, the animals received a daily subcutaneous bolus injection of L-glutamic acid monosodium salt (Sigma, USA) in saline $0.9 \%$ ( $4 \mathrm{mg} / \mathrm{g}$ body mass), in the cervical region, between 7:30 - 9:00 h of light period, at a maximum volume of 0.2 $\mathrm{mL}$ (MSG animals), until they were 10 days old. At 22 days, the animals were weaned; and the female was removed from the cage [21]. At 90 days, obese animals were selected as previously described $[19,20]$. Briefly, the Lee index (body mass $(\mathrm{g})^{0.33} /$ naso-anal length $\left.(\mathrm{cm})\right)[22,23]$ was calculated and these animals treated with L-glutamic acid monosodium salt were then subdivided into 2 groups: MSG (obese $=$ Lee index > 0.300) and MSG-FD (obese fasted for 72 hours). Rats of the same age and strain, receiving $0.9 \% \mathrm{NaCl}$ under the same experimental conditions, were subdivided into 2 groups: $\mathrm{C}$ (normal $=$ Lee index $\leq 0.300=$ control) and FD (normal fasted for 72 hours). Food deprivation was performed by transferring pairs of animals, between 7:30 9:00 h of light period, into metabolic cages, where they were housed without food and with water ad libitum for 72 hours. Except during this period, all experimental groups had access ad libitum to the same ration Nuvilab CR-1.

The animal care and handling procedures used were in accordance with the guidelines of the Brazilian College of Animal Experimentation and were approved by the Ethics Committee of Institute Butantan (291/06).

\section{Brain collection and fixation}

The animals were anesthetized with a solution containing ketamine chlorhydrate (Konig, Argentina) $(100 \mathrm{mg} / \mathrm{mL})$ and xylazine chlorhydrate (Vetbrands, Brazil) $(100 \mathrm{mg} / \mathrm{mL})$ by intraperitoneal injection (ip) $(0.2 \mathrm{~mL} / 100 \mathrm{~g}$ of body mass $)$ between 4:00 - 6:30 $\mathrm{h}$ during the light phase, and subsequently received intracardiac bolus injection of $0.1 \mathrm{~mL}$ of sodium heparin solution (Roche, Brazil) (1000 IU/mL of saline). Cardiac perfusion with $0.9 \% \mathrm{NaCl}$ plus $50 \mathrm{mM}$ phosphate buffer, $\mathrm{pH} 7.4$, over a period of 5 - $10 \mathrm{~min}$, followed by fixative solution composed of 4\% paraformaldehyde (Synth, Brazil) in 4\% sodium borate (borax) (Synth, Brazil), pH 9.5, for another $5-10 \mathrm{~min}$, at a flow rate of $12-15 \mathrm{~mL} / \mathrm{min}$, was performed as described by Alponti et al [20]. After perfusion, the animals were decapitated with a guillotine and the brains were removed for immediate use.

\section{Histological procedures}

The brains were post-fixed in 4\% paraformaldehyde solution containing $20 \%$ sucrose (w/v), for $4 \mathrm{~h}$. After this period, it was cryoprotected in KPBS (potassium phosphate buffered saline, $0.02 \mathrm{M}$, pH 7.4) plus $20 \%$ sucrose for $24 \mathrm{~h}$ and thus sectioned into $40 \mu \mathrm{m}$ thick coronal section (from Bregma -1.32 , in the rostrocaudal axis), using a cryomicrotome (Leica SM 2000R, Leica, Germany). Five series of sections were collected for each animal in acrylic plates (25 wells, TPP, Switzerland) with antifreeze solution (PBS 0.1 M, pH 7.4, plus $20 \%$ sucrose and ethylene glycol) and stored at $-20{ }^{\circ} \mathrm{C}$ until submission to the following procedures.

\section{Immunohistochemistry of CD26 and Fos}

Coronal sections were removed from antifreeze solution and then washed in KPBS ( $2 \times 10 \mathrm{~min}), 0.1 \%$ hydrogen peroxide ( 1 x $10 \mathrm{~min}$ ) and KPBS ( 2 x $10 \mathrm{~min})$. They were then incubated at $18{ }^{\circ} \mathrm{C}$ with polyclonal antisera anti-CD26 of human produced in rabbit (Santa Cruz Biotech., USA) or anti-Fos of human produced in rabbit (Oncogene, USA), for $48 \mathrm{~h}$, in 1.5 $\mathrm{mL}$ microtubes placed in a mixer and protected from light. The primary antibody solutions were composed of KPBS, $0.3 \%$ Triton X-100, 3\% normal goat serum (Vector Laboratories, USA) and anti-CD26 (1: 1,000 in KPBS) or antiFos (1: 10,000 in KPBS). After this incubation, the sections were washed in KPBS ( $2 \times 10 \mathrm{~min})$ and thus incubated at $18{ }^{\circ} \mathrm{C}$ for $60 \mathrm{~min}$ with a solution of KPBS plus $0.3 \%$ Triton X-100 and the secondary antibody (biotinylated goat antirabbit) (1 : 500) (Vector Laboratories, USA). Subsequently, the samples were washed in KPBS ( $2 \times 10 \mathrm{~min})$, incubated at $18^{\circ} \mathrm{C}$ with avidin-biotin-peroxidase complex (1: 500) (ABC Kit Vectastain Elite Standard, Vector Laboratories, USA) for $60 \mathrm{~min}$, washed with $\mathrm{KPBS}(2 \times 10 \mathrm{~min})$ and incubated at 18 ${ }^{\circ} \mathrm{C}$ with a solution containing 3,3-diaminobenzidine (Sigma, USA) $(0.05 \%$ in KPBS $)$, nickel ammonium sulfate $(0.05 \%$ in KPBS) and hydrogen peroxide $(0.01 \%$ in KPBS) (immunoperoxidase reaction). To stop the reaction, the sections were submitted to a final washing with KPBS ( 2 x $10 \mathrm{~min})$. Then the sections were placed on slides previously covered with poly-L-lysine (Sigma, USA).

\section{Nissl stain}

Coronal sections were removed from antifreeze solution and then washed in KPBS ( 2 x 10 min) and placed on slides previously covered with poly-L-lysine. Subsequently, they were dried at $23{ }^{\circ} \mathrm{C}$ for $48 \mathrm{~h}$ and then diaphanized in xilol and dehydrated in ascending concentrations of ethanol. After re- 

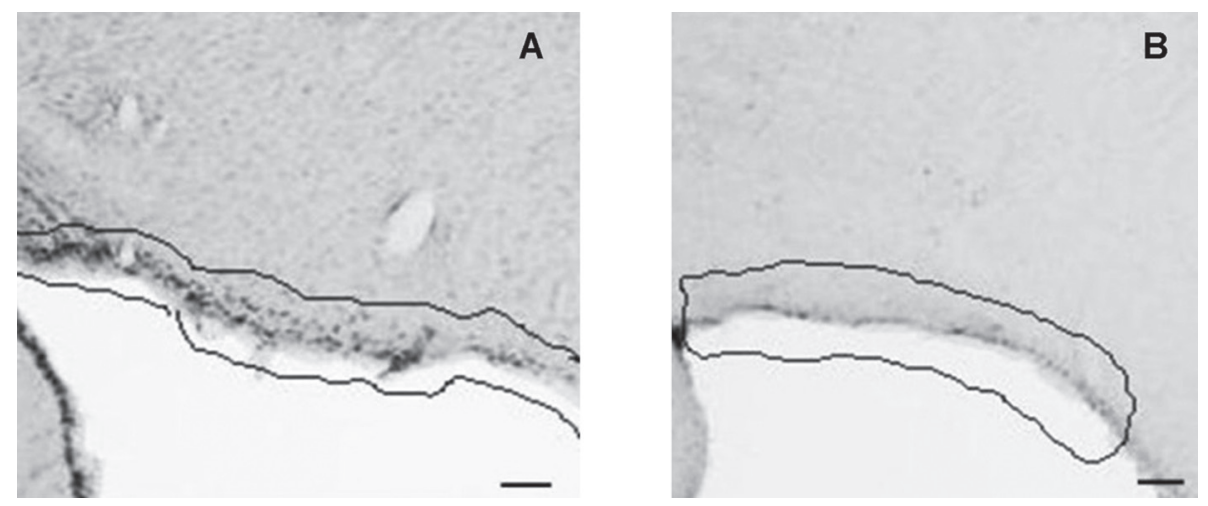

Figure 1. CD26 immunoreactivity in coronal section of rat brain. Pia mater (highlighted) of control (A) and food deprived MSG (B) animals. $2.12 \mathrm{~mm}$ posterior to bregma. Bars $=30 \mu \mathrm{m}$.

hydrated in descending concentrations of ethanol until pure water, the sections were immersed in thionin (Fisher Scientific, USA) for $20 \mathrm{sec}$.

\section{Preparation of slides}

After immunohistochemistry or Nissl stain procedures, the sections were dehydrated in ascending concentrations of ethanol, diaphanized in xilol and coverslipped with coverglass over Eukitt (Electron Microscopy Sciences, USA).

\section{Microdensitometry of CD26 and Fos immunoreactivity}

The capture of images and microdensitometry analyses (populational density) of coronal sections were carried out with Nikon E-1000 microscope (Nikon Corporation, Japan), equipped with a digital camera CoolSNAP ${ }^{\circledR}$, using ImagePro-Plus $4.0^{\circledR}$ (Media Cybernetics) software. The photo-

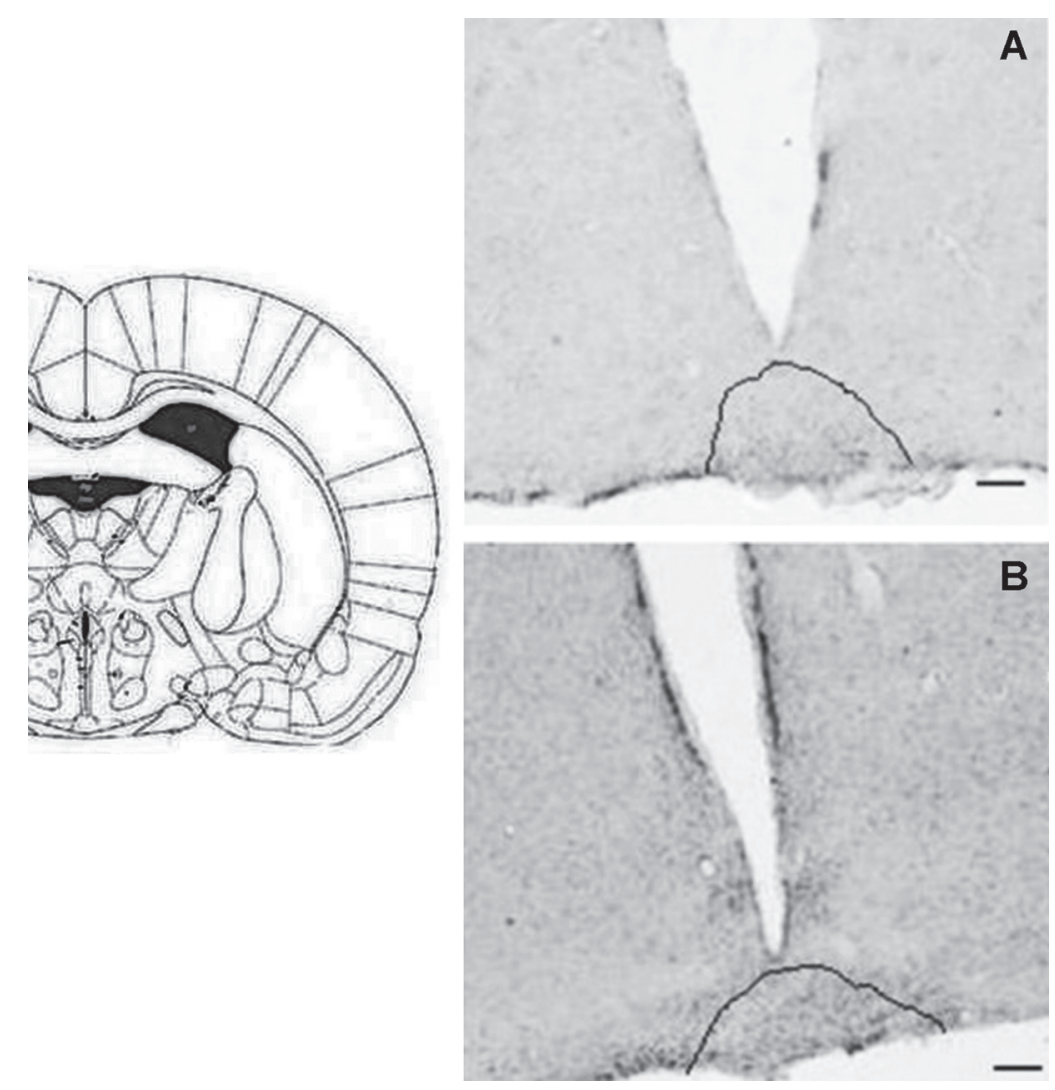

Figure 2. CD26 immunoreactivity in coronal section of rat brain. Retrochiasmatic nuclei (highlighted) of control (A) and MSG (B) animals. $1.44 \mathrm{~mm}$ posterior to bregma. Bars $=30 \mu \mathrm{m}$. 
Table 1. Density of CD26-ir in the Hypothalamus of Normal Control (C), Normal Food Deprived (FD), Obese (MSG) and Obese Food Deprived (MSG-FD) Animals

\begin{tabular}{lllll}
\hline Regions & C & FD & MSG & MSG-FD \\
\hline ArcNH & $306 \pm 11.26$ & $490 \pm 33.77^{* *}$ & $144 \pm 15.88^{* *}$ & $292 \pm 26.56$ \\
$(1.71-4.36 \mathrm{~mm}) \#$ & $(3)$ & $(3)$ & $(3)$ & $(3)$ \\
DMH & $14 \pm 0.88$ & $27 \pm 1.45^{* *}$ & $10 \pm 1.15$ & $\mathrm{~A}$ \\
$(2.28-3.84 \mathrm{~mm}) \#$ & $(3)$ & $(3)$ & $(3)$ & $(3)$ \\
Pe & $63 \pm 12.12$ & $134 \pm 19.63^{*}$ & $52 \pm 9.81$ & $144 \pm 24.26^{*}$ \\
$(1.32-3.48 \mathrm{~mm}) \#$ & $(3)$ & $(3)$ & $(3)$ & $(3)$ \\
PM & $425 \pm 37.58$ & $58 \pm 7.22^{* * *}$ & $256 \pm 20.71^{*}$ & $51 \pm 5.19^{* * *}$ \\
$(1.32-4.36 \mathrm{~mm}) \#$ & $(3)$ & $(3)$ & $(3)$ & $(3)$ \\
PVN & $47 \pm 4.91$ & $88 \pm 6.93^{* *}$ & $\mathrm{~A}$ & $\mathrm{~A}$ \\
$(1.32-2.28 \mathrm{~mm}) \#$ & $(3)$ & $(3)$ & $(3)$ & $(3)$ \\
RCh & $12 \pm 1.15$ & $41 \pm 7.8^{*}$ & $138 \pm 11.14^{* * *}$ & $\mathrm{~A}$ \\
$(1.32-2.52 \mathrm{~mm}) \#$ & $(3)$ & $(3)$ & $(3)$ & $(3)$ \\
SON & $8 \pm 1.45$ & $\mathrm{~A}$ & $79 \pm 1.45^{* * *}$ & $\mathrm{~A}$ \\
$(1.32-2.52 \mathrm{~mm}) \#$ & $(3)$ & $(3)$ & $(3)$ & $(3)$ \\
\hline
\end{tabular}

Values are mean \pm SEM. Number of animals in the parentheses. $A=$ absence of immunoreactivity. ${ }^{*} P<0.05,{ }^{* *} P<$ 0.009 and ${ }^{* * *} \mathrm{P}<0.0001$ compared to $\mathrm{C}$ (paired, two-sided Student's t test). ArcNH: arcuate nucleus hypothalamic; $\mathrm{DMH}$ : dorsomedial hypothalamus; Pe: periventricular hypothalamic nucleus; PVN: paraventricular hypothalamic nucleus; RCh: retrochiasmatic hypothalamic nucleus; SON: supraoptic hypothalamic nucleus; PM: pia mater. \#Reference of localization in $\mathrm{mm}$ posterior to the bregma [24].

graphs were taken with a digital camera CoolSNAP ${ }^{\circledR}$ (Nikon Corporation, Japan). The quantitative results of CD26 and Fos expression are related to the frequency of stained structures and not the absolute concentrations of these compounds. The analyzed areas from which the frequency of stained structures were obtained were in accordance with the atlas of the rat brain [24].

\section{Data analysis}

The quantitative data were presented as mean \pm standard error of mean (SEM) and statistically analyzed using GraphPad Instat ${ }^{\mathrm{TM}}$, GraphPad Prism ${ }^{\mathrm{TM}}$ and Prism 3.0 software packages. The Student's t test was used to compare pairs of values. In all calculations a minimum critical level of $\mathrm{P}<$ 0.05 was set.

\section{Results}

Nissl staining confirmed the injury of ArcNH and ME, typical of MSG-obesity model (data not shown).

$\mathrm{C}$ animals had CD26 immunoreactivity (CD26-ir) in the following HT nuclei: arcuate (ArcNH), dorsomedial $(\mathrm{DMH})$, periventricular $(\mathrm{Pe})$, paraventricular $(\mathrm{PVN})$, retrochiasmatic ( $\mathrm{RCh}$ ) and supraoptic (SON). CD26-ir was also detected in the pia mater (PM). Figures 1 and 2 illustrate the areas where significant changes of CD26-ir were detected, compared to $\mathrm{C}$ animals. Table 1 shows the density of CD26ir in representative animals of each experimental condition.
CD26-ir increased in the ArcNH (490 \pm 33.77$)$, DMH (27 \pm $1.45), \mathrm{Pe}(134 \pm 19.63), \mathrm{PVN}(88 \pm 6.93)$ and $\mathrm{RCh}(41 \pm 7.8)$ and decreased in the PM $(58 \pm 7.22)$ of FD animals, compared to C (ArcNH: $306 \pm 11.26$; DMH: $14 \pm 0.88$; Pe: $63 \pm$ 12.12; PM: $425 \pm 37.58$; PVN: $47 \pm 4.91$; RCh: $12 \pm 1.15$ ). In FD animals, CD26-ir was absent in the SON. CD26-ir of MSG animals increased in the RCh $(138 \pm 11.14)$ and SON $(79 \pm 1.45)$, and decreased in the ArcNH $(144 \pm 15.88)$ and PM $(256 \pm 20.71)$, compared to C (ArcNH: $306 \pm 11.26$; PM: $425 \pm 37.58$; RCh: $12 \pm 1.15$; SON: $8 \pm 1.45$ ). CD26-ir was absent in the PVN in MSG animals. CD26-ir of MSGFD decreased in PM $(51 \pm 5.19)$ and increased in Pe (144 \pm 24.26), compared to $\mathrm{C}$ (Pe: $63 \pm 12.12$; PM: $425 \pm 37.58$ ). The absence of CD26-ir was observed in DMH, PVN, RCh and SON of MSG-FD.

Immunoreactivity to Fos (Fos-ir) was detected in the following HT nuclei of $\mathrm{C}$ animals: anterior $(\mathrm{AH})$ and ventromedial (VMH) hypothalamus, ArcNH, DMH, Pe, PVN, RCh and SON.

Table 2 summarizes the mean density of Fos-ir in each experimental group. Fos-ir decreased in the PVN of FD (135 $\pm 14.14)$, MSG $(66 \pm 7.50)$ and MSG-FD $(30 \pm 4.61)$ and in the RCh of FD $(65 \pm 7.50)$, MSG $(22 \pm 4.04)$ and MSG-FD (66 \pm 11.26 ), compared to C (PVN: $207 \pm 16.16$; RCh: 155 $\pm 19.92)$. Fos-ir decreased in the VMH of FD $(74 \pm 12.70)$ and MSG-FD $(118 \pm 21.94)$, compared to C $(408 \pm 79.67)$. Fos-ir of MSG decreased in the AH, DMH and Pe (AH: 27 \pm 4.91 ; DMH: $12 \pm 2.31$; Pe: $16 \pm 1.15)$, while it decreased in ArcNH (101 \pm 23.67$)$, DMH $(239 \pm 41.28)$ and Pe (16 \pm 2.03) of MSG-FD, compared to C (AH: $63 \pm 11.83$; ArcNH: 
Table 2. Density of Fos-ir in the Hypothalamus of Normal Control (C), Normal Food Deprived (FD), Obese (MSG) and Obese Food Deprived (MSG-FD) Animals

\begin{tabular}{lllll}
\hline Regions & C & FD & MSG & MSG-FD \\
\hline AH & $63 \pm 11.83$ & $51 \pm 9.52$ & $27 \pm 4.91^{*}$ & $60 \pm 6.64$ \\
$(1.32-2.82) \#$ & $(3)$ & $(3)$ & $(3)$ & $(3)$ \\
ArcNH & $437 \pm 66.11$ & $352 \pm 31.46$ & $347 \pm 61.77$ & $101 \pm 23.67 * *$ \\
$(1.71-4.36) \#$ & $(3)$ & $(3)$ & $(3)$ & $(3)$ \\
DMH & $577 \pm 111.14$ & $443 \pm 69$ & $12 \pm 2.31^{* *}$ & $239 \pm 41.28^{*}$ \\
$(2.28-3.84) \#$ & $(3)$ & $(3)$ & $(3)$ & $(3)$ \\
Pe & $120 \pm 16.45$ & $78 \pm 4.91$ & $16 \pm 1.15^{* *}$ & $16 \pm 2.03^{* *}$ \\
$(1.32-3.48) \#$ & $(3)$ & $(3)$ & $(3)$ & $(3)$ \\
PVN & $207 \pm 16.16$ & $135 \pm 14.14^{*}$ & $66 \pm 7.50^{* *}$ & $30 \pm 4.61^{* * *}$ \\
$(1.32-2.28) \#$ & $(3)$ & $(3)$ & $(3)$ & $(3)$ \\
RCh & $155 \pm 19.92$ & $65 \pm 7.50^{*}$ & $22 \pm 4.04^{* *}$ & $66 \pm 11.26^{*}$ \\
$(1.32-2.52) \#$ & $(3)$ & $(3)$ & $(3)$ & $(3)$ \\
SON & $25 \pm 2.60$ & $\mathrm{~A}$ & $\mathrm{~A}$ & $\mathrm{~A}$ \\
$(1.32-2.52) \#$ & $(3)$ & $(3)$ & $(3)$ & $(3)$ \\
VMH & $408 \pm 79.67$ & $74 \pm 12.70^{*}$ & $\mathrm{~A}$ & $118 \pm 21.94 *$ \\
$(1.72-3.36) \#$ & $(3)$ & $(3)$ & $(3)$ & $(3)$ \\
\hline
\end{tabular}

Values are mean \pm SEM. Number of animals in the parentheses. $A=$ absence of immunoreactivity. ${ }^{*} P$ $<0.05$, ${ }^{* *} \mathrm{P}<0.009$ and ${ }^{* *} \mathrm{P}<0.0006$ compared to $\mathrm{C}$ (paired, two-sided Student's $\mathrm{t}$ test). AH: anterior hypothalamus; ArcNH: arcuate hypothalamic nucleus; $\mathrm{DMH}$ : dorsomedial hypothalamus; Pe: periventricular hypothalamic nucleus; PVN: paraventricular hypothalamic nucleus; RCh: retrochiasmatic hypothalamic nucleus; SON: supraoptic hypothalamic nucleus; VMH: ventromedial hypothalamus. \#Reference of localization in $\mathrm{mm}$ posterior to the bregma [24].

$437 \pm 66.11$; DMH: $577 \pm 111.14$; Pe: $120 \pm 16.45$ ). Fos-ir was absent in the SON of FD, MSG and MSG-FD animals, as well as in the VMH of MSG animals.

\section{Discussion}

DPPIV enzyme activity in blood plasma [19] and hypothalamus [20] was previously distinguished kinetically as insensitive (DI) (identified as CD26 protein) and sensitive (DS) to diprotin A. It was reported that MSG and/or FD decreased the activity of soluble and membrane-bound hypothalamic DPPIV-DI, as well as the activity of soluble hypothalamic DPPIV-DS [20]. Furthermore, the monoclonal protein expression of membrane-bound CD26 also decreased in the hypothalamus of MSG and/or FD [20]. Despite of the increasing relevance of CD26 in recent years, due to the therapeutic role of the inhibitors of DPPIV activity [15] and DPPIV-resistant GLP-1 agonist exenatide [25] in diabetes mellitus type 2 , until now the data from that descriptive study [20] were the unique about the involvement of CD26/DPPIV in the regulation of the energy balance in the CNS. The present study added the mapping of CD26 in the arcuate, dorsomedial, periventricular, retrochiasmatic and supraoptic hypothalamic nuclei, which allows us a first step to understand the association of the presence and altered levels of this molecule with specific physiological roles of each nucleus. Among these nuclei the presence of CD26 in the arcuate nucleus seems to be the most impressive due to the well-known role of this nucleus in the energy metabolism $[26,27]$ and since the synthesis of NPY 1-36 is modulated by receptors for insulin and leptin in this nucleus [26-30]. It was also known that under food deprivation the increase of orexigenic peptides occurs, such as opioids and NPY 1-36 (both are DPPIV substrates) [31-35], which may be related to the observed increase in CD26-ir in these hypothalamic nuclei under food deprivation (except in supraoptic nucleus). However, the MSG obese animals had a decreased CD26-ir in the arcuate nucleus, which may be resulting from the specific damage caused in this nucleus by MSG treatment [3638] as confirmed here by Nissl staining. However, increased CD26-ir was observed in MSG in the supraoptic nucleus, which synthesizes vasopressin (AVP). The content of NPY 1-36 is known to be high in obese [39] and it modifies the release of AVP [40], justifying the increase of CD26-ir cell bodies in this nucleus. In the periventricular hypothalamic nucleus CD26-ir also increased in normal and MSG animals both under food deprivation. This nucleus has neurons in contact with cerebrospinal fluid and thus it is sensitive to changes in the composition of this fluid. In the retrochiasmatic nucleus, CD26-ir was higher in normal food deprived and MSG, and lower in food deprived MSG animals. Since this nucleus expresses POMC mRNA [41] and DPPIV hydrolyzes $\beta$-endorphin, derived from POMC [42], it is likely that the increase of CD26-ir in this nucleus is related to DPPIV activity of CD26. The immunohistochemical analysis 
also showed that CD26-ir in the dorsomedial hypothalamic nucleus increased in normal food deprived and decreased in MSG animals submitted or not to food deprivation. This nucleus has an integrative function because it receives projections from several other hypothalamic nuclei, especially the arcuate nucleus $[30,43]$. Thus, the increase of CD26-ir observed in normal food deprived suggests that CD26 acts in the control of orexigenic peptides, such as NPY 1-36. On the other hand, the damage caused by MSG in the arcuate nucleus of obese animals probably also caused the decrease of CD26-ir in the dorsomedial nucleus, as a result of reduced projections from the arcuate nucleus. Similarly, there is a decrease in CD26-ir in the paraventricular nucleus of MSG animals submitted or not to food deprivation. This nucleus also receives projections from the arcuate nucleus. It was noteworthy the presence of CD26-ir cell bodies in the pia mater, which is known to be rich in astrocytes [44]. CD26-ir was reduced in this meninx of normal food deprived and MSG animals submitted or not to food deprivation. The astrocytes are important glial cells which have several functions, including regulation of neurotransmitters through the membrane-bound transporter proteins that remove neurotransmitters from synaptic cleft, such as glutamate transporters [45].

Fos-ir is known to be increased in the supramammillary and dorsomedial hypothalamic nuclei of obese mice and in the medial part of perifornical nucleus of lean mice, after food deprivation [46]. In the present study, Fos-ir decreased in the dorsomedial nucleus of MSG animals submitted or not to food deprivation. These opposite results can also be explained by the lesions of arcuate nucleus caused by the adopted experimental model, as mentioned before. Moreover, it was reported that a diet of saturated fat modulates the hypothalamic neuronal activity (measured by Fos-ir) in a pattern consistent with its obesogenic effects [47]. The results of the present study evidenced a decrease of Fos-ir in the ventromedial nucleus of normal food deprived and MSG animals submitted or not to food deprivation, which can be hypothesized as due to unbalanced satiety in these animal groups, since the ventromedial nucleus is known as the satiety center in the CNS. Overall, reduced activity of the arcuate nucleus in the animals treated with MSG may lead to decreased production of orexigenic and anorexigenic peptides. Furthermore, the paraventricular, retrochiasmatic and supraoptic nuclei also had decreased cell activity (Fos-ir) in normal food deprived, MSG and food deprived MSG animals, which may implicate in impaired production of orexigenic and anorexigenic peptides in these nuclei. In this study, changes on the levels of CD26 and Fos-ir had the same sense (both declined) in the dorsomedial nucleus of food deprived MSG, the paraventricular nucleus of MSG and food deprived MSG, the retrochiasmatic nucleus of the food deprived MSG and in the supraoptic nucleus of normal food deprived and food deprived MSG, which could be linked with a reduced production of CD26 under these situations in these brain areas. However,
Fos-ir and CD26-ir had opposite changes in the retrochiasmatic nucleus of normal food deprived and MSG, the arcuate nucleus of normal food deprived, MSG and food deprived MSG, the periventricular nucleus of normal food deprived, MSG and food deprived MSG, the paraventricular nucleus of normal food deprived, the supraoptic nucleus of MSG and in the dorsomedial nucleus of normal food deprived. Therefore, physiological significance of a possible association between changes on CD26-ir and Fos-ir in the same brain areas is difficult to interpret. However, they permit to select these areas to further identification of possible cellular targets that regulate CD26 in the examined situations.

In conclusion, our data provide clear evidences for regional regulation of CD26 expression in certain areas of the hypothalamus of MSG obese and fasting rats, which are coupled with changes on cellular activity. The topographical distribution of this multifunctional CD26 protein in the hypothalamus suggests that its regulation is a hallmark of hypothalamic exposure to metabolic challenges.

\section{Acknowledgements}

We would like to sincerely thank all the staff at Laboratory of Pharmacology for technical support and constructive comments on this manuscript.

\section{Grant}

This investigation was supported by a Research Grant 05/04699-2 from FAPESP (Fundacao de Amparo a Pesquisa do Estado de Sao Paulo, Brazil). P.F.S. was recipient of a CNPq (Conselho Nacional de Desenvolvimento Cientifico e Tecnologico, Brazil) productivity grant. R.F.A. was recipient of a CAPES (Coordenacao de Aperfeicoamento de Pessoal de Nivel Superior, Brazil) fellowship.

\section{References}

1. Fleischer B. CD26: a surface protease involved in T-cell activation. Immunol Today 1994;15(4):180-184.

2. Steinbrecher A, Reinhold D, Quigley L, Gado A, Tresser N, Izikson L, Born I, et al. Targeting dipeptidyl peptidase IV (CD26) suppresses autoimmune encephalomyelitis and up-regulates TGF-beta 1 secretion in vivo. J Immunol 2001;166(3):2041-2048.

3. Williams YN, Baba H, Hayashi S, Ikai H, Sugita T, Tanaka S, Miyasaka N, et al. Dipeptidyl peptidase IV on activated T cells as a target molecule for therapy of rheumatoid arthritis. Clin Exp Immunol 2003;131(1):68-74.

4. Bjelke JR, Christensen J, Nielsen PF, Branner S, Kan- 
strup AB, Wagtmann N, Rasmussen HB. Dipeptidyl peptidases 8 and 9: specificity and molecular characterization compared with dipeptidyl peptidase IV. Biochem J 2006;396(2):391-399.

5. Unniappan S, McIntosh $\mathrm{CH}$, Demuth HU, Heiser U, Wolf R, Kieffer TJ. Effects of dipeptidyl peptidase IV on the satiety actions of peptide YY. Diabetologia 2006;49(8):1915-1923.

6. Medeiros MD, Turner AJ. Processing and metabolism of peptide-YY: pivotal roles of dipeptidylpeptidase-IV, aminopeptidase-P, and endopeptidase-24.11. Endocrinology 1994;134(5):2088-2094.

7. Mentlein R. Dipeptidyl-peptidase IV (CD26)--role in the inactivation of regulatory peptides. Regul Pept 1999;85(1):9-24.

8. Ghersi G, Chen W, Lee EW, Zukowska Z. Critical role of dipeptidyl peptidase IV in neuropeptide Y-mediated endothelial cell migration in response to wounding. Peptides 2001;22(3):453-458.

9. Batterham RL, Cowley MA, Small CJ, Herzog H, Cohen MA, Dakin CL, Wren AM, et al. Gut hormone PYY(3-36) physiologically inhibits food intake. Nature 2002;418(6898):650-654.

10. Gabrilovac J, Abramic M, Uzarevic B, Andreis A, Poljak L. Dipeptidyl peptidase IV (DPPIV) enzyme activity on immature T-cell line R1.1 is down-regulated by dynorphin-A(1-17) as a non-substrate inhibitor. Life Sci 2003;73(2):151-166.

11. Sakurada C, Sakurada S, Hayashi T, Katsuyama S, TanNo K, Sakurada T. Degradation of endomorphin-2 at the supraspinal level in mice is initiated by dipeptidyl peptidase IV: an in vitro and in vivo study. Biochem Pharmacol 2003;66(4):653-661.

12. Sakurada C. [Development of a new analgesic based on metabolism of endomorphin, an endogenous opioid peptide]. Yakugaku Zasshi 2004;124(8):549-554.

13. Gorrell MD. Dipeptidyl peptidase IV and related enzymes in cell biology and liver disorders. Clin Sci (Lond) 2005;108(4):277-292.

14. Mantovani A, Gray PA, Van Damme J, Sozzani S. Macrophage-derived chemokine (MDC). J Leukoc Biol 2000;68(3):400-404.

15. Idris I, Donnelly R. Dipeptidyl peptidase-IV inhibitors: a major new class of oral antidiabetic drug. Diabetes Obes Metab 2007;9(2):153-165.

16. Wajchenberg BL. Postprandial glycemia and cardiovascular disease in diabetes mellitus. Arq Bras Endocrinol Metabol 2007;51(2):212-221.

17. Knauf C, Cani PD, Kim DH, Iglesias MA, Chabo C, Waget A, Colom A, et al. Role of central nervous system glucagon-like Peptide-1 receptors in enteric glucose sensing. Diabetes 2008;57(10):2603-2612.

18. Reinhold D, Bank U, Tager M, Ansorge S, Wrenger S, Thielitz A, Lendeckel U, et al. DP IV/CD26, APN/CD13 and related enzymes as regulators of $\mathrm{T}$ cell immunity: implications for experimental encephalomyelitis and multiple sclerosis. Front Biosci 2008;13:2356-2363.

19. Alponti RF, Silveira PF. Neutral aminopeptidase and dipeptidyl peptidase IV activities in plasma of monosodium glutamate obese and food-deprived rats. Obesity (Silver Spring) 2010;18(7):1312-1317.

20. Alponti RF, Frezzatti R, Barone JM, Alegre Vde S, Silveira PF. Dipeptidyl peptidase IV in the hypothalamus and hippocampus of monosodium glutamate obese and food-deprived rats. Metabolism 2011;60(2):234-242.

21. Kaufhold A, Nigam PK, Dhir RN, Shapiro BH. Prevention of latently expressed CYP2C11, CYP3A2, and growth hormone defects in neonatally monosodium glutamate-treated male rats by the N-methyl-D-aspartate receptor antagonist dizocilpine maleate. J Pharmacol Exp Ther 2002;302(2):490-496.

22. Bernardis LL, Patterson BD. Correlation between 'Lee index' and carcass fat content in weanling and adult female rats with hypothalamic lesions. J Endocrinol 1968;40(4):527-528.

23. Rogers P, Webb GP. Estimation of body fat in normal and obese mice. Br J Nutr 1980;43(1):83-86.

24. Paxinos G, Watson C. The rat brain in stereotaxic coordinates. 6th ed. Amsterdam: Academic Press, 2007.

25. Aroda VR, Ratner R. The Safety and Tolerability of GLP-1 Receptor Agonists in the Treatment of Type 2 Diabetes: A Review. Diabetes Metab Res Rev 2011.

26. Arora S, Anubhuti. Role of neuropeptides in appetite regulation and obesity--a review. Neuropeptides 2006;40(6):375-401.

27. van Dijk G, Evers SS, Guidotti S, Thornton SN, Scheurink AJ, Nyakas C. The lateral hypothalamus: A site for integration of nutrient and fluid balance. Behav Brain Res 2011;221(2):481-487.

28. Ghamari-Langroudi M, Cone RD. Shining a light on energy homeostasis. Cell Metab 2011;13(3):235-236.

29. Zigman JM, Elmquist JK. Minireview: From anorexia to obesity--the yin and yang of body weight control. Endocrinology 2003;144(9):3749-3756.

30. Wynne K, Stanley S, McGowan B, Bloom S. Appetite control. J Endocrinol 2005;184(2):291-318.

31. Iwasa T, Matsuzaki T, Kinouchi R, Gereltsetseg G, Murakami $\mathrm{M}$, Nakazawa $\mathrm{H}$, Yasui $\mathrm{T}$, et al. Changes in the responsiveness of serum leptin and hypothalamic neuropeptide Y mRNA levels to food deprivation in developing rats. Int J Dev Neurosci 2011;29(4):377-380.

32. Beck B. Neuropeptide $Y$ in normal eating and in genetic and dietary-induced obesity. Philos Trans R Soc Lond B Biol Sci 2006;361(1471):1159-1185.

33. Moran TH. Gut peptide signaling in the controls of food intake. Obesity (Silver Spring) 2006;14 Suppl 5:250S$253 \mathrm{~S}$.

34. Crane C, Akhter N, Johnson BW, Iruthayanathan M, 
Syed F, Kudo A, Zhou YH, et al. Fasting and glucose effects on pituitary leptin expression: is leptin a local signal for nutrient status? J Histochem Cytochem 2007;55(10):1059-1073.

35. Sucajtys-Szulc E, Goyke E, Korczynska J, Stelmanska E, Rutkowski B, Swierczynski J. Chronic food restriction differentially affects NPY mRNA level in neurons of the hypothalamus and in neurons that innervate liver. Neurosci Lett 2008;433(3):174-177.

36. Pelz KM, Routman D, Driscoll JR, Kriegsfeld LJ, Dark J. Monosodium glutamate-induced arcuate nucleus damage affects both natural torpor and 2DG-induced torporlike hypothermia in Siberian hamsters. Am J Physiol Regul Integr Comp Physiol 2008;294(1):R255-265.

37. Leigh FS, Kaufman LN, Young JB. Diminished epinephrine excretion in genetically obese $(\mathrm{ob} / \mathrm{ob})$ mice and monosodium glutamate-treated rats. Int J Obes Relat Metab Disord 1992;16(8):597-604.

38. Dolnikoff M, Martin-Hidalgo A, Machado UF, Lima FB, Herrera E. Decreased lipolysis and enhanced glycerol and glucose utilization by adipose tissue prior to development of obesity in monosodium glutamate (MSG) treated-rats. Int $\mathrm{J}$ Obes Relat Metab Disord 2001;25(3):426-433.

39. Frerker N, Wagner L, Wolf R, Heiser U, Hoffmann T, Rahfeld JU, Schade J, et al. Neuropeptide Y (NPY) cleaving enzymes: structural and functional homologues of dipeptidyl peptidase 4. Peptides 2007;28(2):257-268.
40. Leibowitz SF. Brain neuropeptide Y: an integrator of endocrine, metabolic and behavioral processes. Brain Res Bull 1991;27(3-4):333-337.

41. Elmquist JK. Hypothalamic pathways underlying the endocrine, autonomic, and behavioral effects of leptin. Physiol Behav 2001;74(4-5):703-708.

42. Furuhashi M, Mizutani S, Kurauchi O, Kasugai M, Narita $\mathrm{O}$, Tomoda $\mathrm{Y}$. In vitro degradation of opioid peptides by human placental aminopeptidase M. Exp Clin Endocrinol 1988;92(2):235-237.

43. Bernardis LL, Bellinger LL. The dorsomedial hypothalamic nucleus revisited: 1998 update. Proc Soc Exp Biol Med 1998;218(4):284-306.

44. Roelofs RF, Fischer DF, Houtman SH, Sluijs JA, Van Haren W, Van Leeuwen FW, Hol EM. Adult human subventricular, subgranular, and subpial zones contain astrocytes with a specialized intermediate filament cytoskeleton. Glia 2005;52(4):289-300.

45. Lee A, Rayfield A, Hryciw DH, Ma TA, Wang D, Pow $\mathrm{D}$, Broer $\mathrm{S}$, et al. $\mathrm{Na}+-\mathrm{H}+$ exchanger regulatory factor 1 is a PDZ scaffold for the astroglial glutamate transporter GLAST. Glia 2007;55(2):119-129.

46. Huang XF, Wang H. Altered c-fos expression in autonomic regulatory centers of genetically obese (ob/ob) mouse brain. Brain Res 1998;799(2):307-310.

47. Wang H, Storlien LH, Huang XF. Influence of dietary fats on c-Fos-like immunoreactivity in mouse hypothalamus. Brain Res 1999;843(1-2):184-192. 